\title{
Defoamers in the Coatings Industry
}

\author{
Albert Frank* and Wilfried Scholz
}

\begin{abstract}
Foam bubbles can be a very severe problem in all kinds of coating systems. Mechanisms of foam stabilization and mechanisms of defoamers are briefly reviewed. The chemistry of different defoamer classes (mineral oil defoamers, silicone defoamers, silicone-free polymeric defoamers) together with their typical application areas are discussed. Information about defoamer selection and evaluation methods is given.
\end{abstract}

Keywords: Defoamer · Foam · Lamella · Mineral oil · Silicone defoamer

\section{Introduction}

Foam is always undesirable in coatings. On the one hand, foam can occur in the paint production process itself, thus leading to non-optimal filling of the production vessels. On the other hand - and this is where most problems arise - foam can also occur during application, thus causing surface defects. Of course, foam functions not only as an optical disturbance, but also as a hindrance to the proper development of the protective function of the coating. Accordingly, defoamers are an essential ingredient in nearly all formulations.

Nearly all paint system components can affect foam behavior either positively or negatively. In addition, the substrate and the application method can have an influence as well. One must realize that foam removal is very much situation specific and a coating material cannot be defoamed 'in general'. For instance, a particular spray application may result in excellent film properties; nevertheless, the utilization of the exact same paint system in a curtain coater operation may create foam problems.

\section{Foam Stabilization}

In liquid coatings foam is defined as a fine distribution of a gas (normally air) in the liquid phase. A characteristic feature of foam (as compared to other physical states) is the extremely large interface between the gas and the liquid and so foam represents a high-energy state of the liquid/gas system.

Directly after formation, the gas bubbles rise through the liquid to reach the surface (Fig. 1). According to Stokes' law, the rate of rise is dependent upon the radius ( $r$ ) of the bubbles and upon the vis$\operatorname{cosity}(\eta)$ of the liquid. When a typical gas bubble reaches the surface, then the liquid which forms the lamella (the thin liquid film surrounding the gas bubble) starts to flow out of the foam lamella. This flow process, which reduces the thickness of the lamella, is denoted as the 'drainage effect' and is the reason for the drying of the foam (Fig. 2).

Drying also changes the structure of the foam: immediately after foam formation, the foam bubbles contain an abun- dance of liquid. This state is known as 'wet foam' or 'spherical foam' because the bubbles are still spherically shaped and do not, to any appreciable extent, deform one another (Fig. 3). When liquid now flows out of the foam lamella (drainage effect) the lamella becomes thinner; the gas bubbles pack more densely together and form polyhedral structures. The resultant foam state is denoted as 'dry' or 'polyhedral' foam. In the absence of opposing effects, the above drainage effect would eventually reduce lamella thickness to the point where the foam would simply collapse. Below approximately $10 \mathrm{~nm}$ thickness the lamella loses its integrity and the foam bubble breaks. This, however, is only the case with pure liquids; pure liquids therefore do not foam.

Foam bubbles are only stable when foam-stabilizing substances are present in the liquid phase. In general, all kinds of interfacially active substances can stabilize foam. Due to the presence of hydrophobic (non-polar) and hydrophilic (polar) segments in the molecular struc-

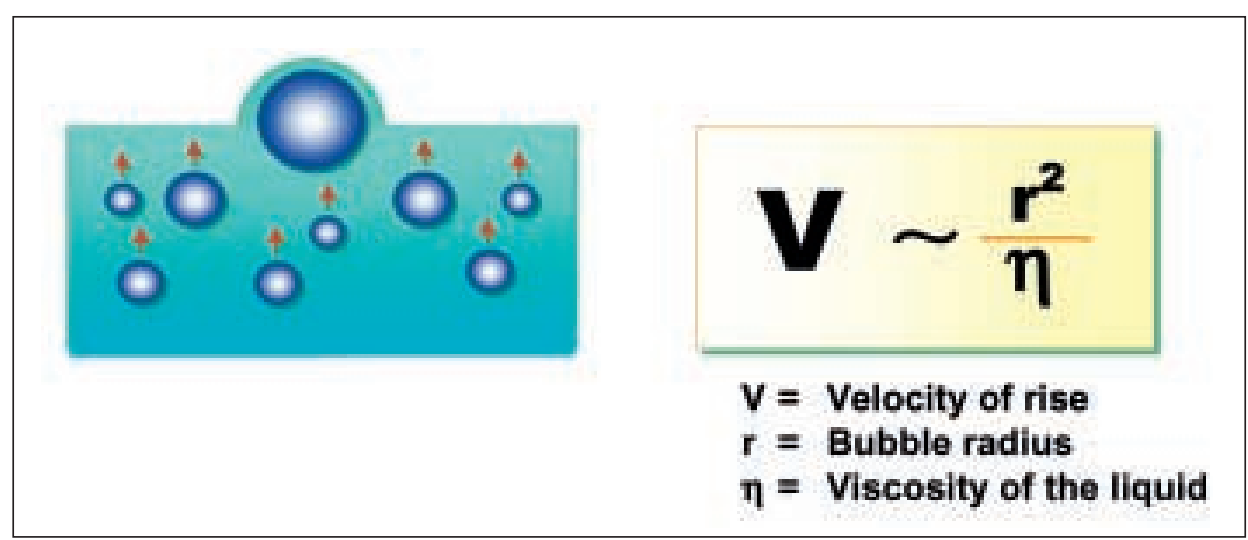

Fig. 1. Gas bubbles rise to the surface of the liquid (Stokes law) 

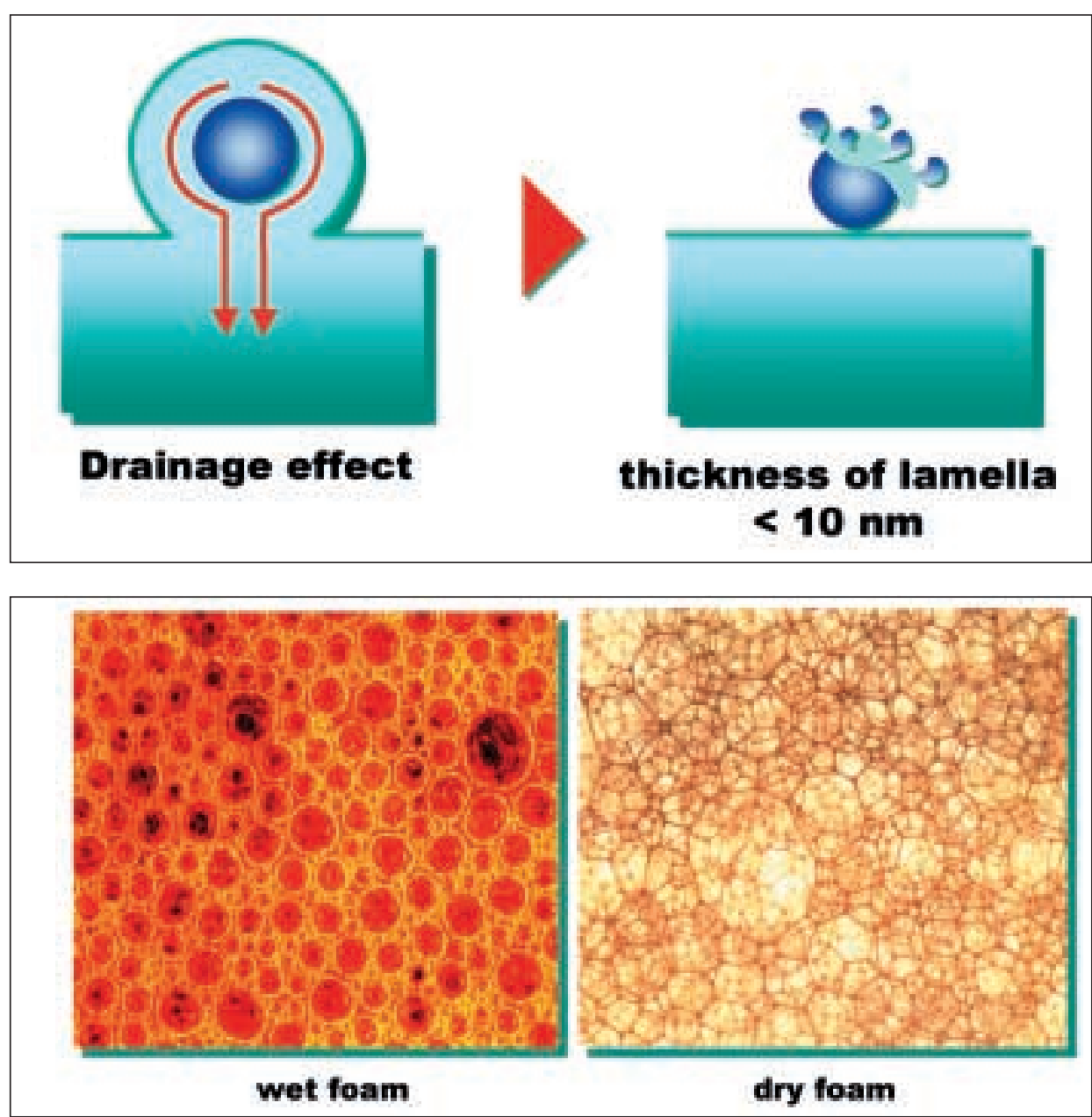

ture of such substances they orient themselves toward the liquid/gas interface. The flow of the liquid out of the lamella is stopped and the lamella will not get thin enough to break. Stopping the drainage process effectively stabilizes the foam bubbles (Fig. 4). Every paint formulation (aqueous, solvent-free, or solvent-based) contains a multitude of potentially foamstabilizing substances of varying origin and chemical structure. Consequently, every formulation may, in principle, develop stable foam bubbles.

\section{Defoaming by Additives}

Since it is virtually impossible to escape the occurrence of potential foamstabilizing substances in coatings systems, defoamers are employed to avoid the formation of foam and/or to destroy (as rapidly as possible) foam which has already been formed.

Fig. 4. Stabilization of foam bubble by surfactants
When the entering coefficient is positive, the defoamer can, of course, enter
Defoamers are low surface tension defoamed

- Positive entering coefficient

- Positive spreading coefficient
Fig. 3. Structure of wet foam (spherical foam)

the foam lamella. If, in addition, the spreading coefficient is positive, then the defoamer product can now actually spread in the interface. Because of this spreading effect, the foam stabilizing surfactants are pushed away, and the previously elastic, disturbance-resisting lamella is replaced by a lamella film which demonstrates both lower surface tension and reduced cohesive forces (Fig. 5).
Fig. 2. Drying of foam bubble (drainage effect) and dry foam (polyhedral foam)

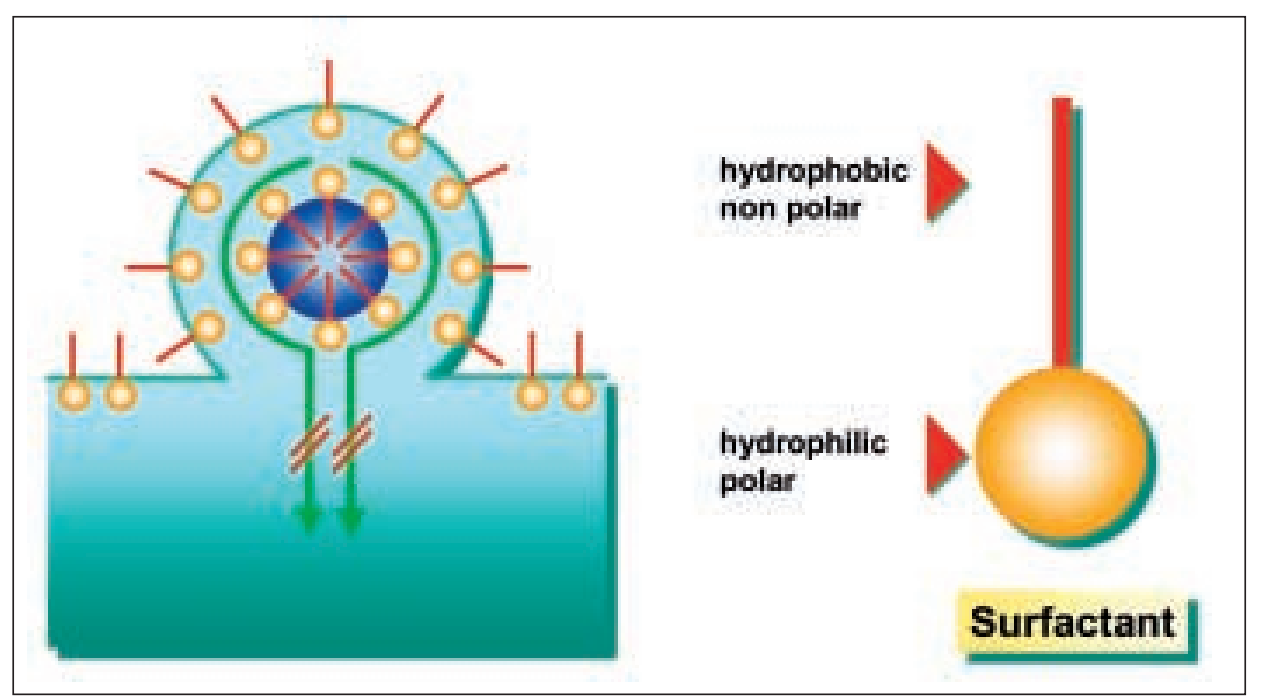


The defoaming mechanism of such defoamer liquids can be accentuated (especially in aqueous systems) by the addition of finely dispersed hydrophobic particles. The defoamer liquid serves as a carrier medium that transports the particles into the foam lamella. On one hand, the hydrophobic particles function in the hydrophilic liquid lamella as 'foreign' particles and therefore contribute to foam destabilization through the reduction of cohesive forces. On the other hand, such particles adsorb or 'capture' surfactant molecules on their surfaces, thereby allowing the foam lamella to collapse (Fig. 6).

Important criteria of all defoamers are their 'selective incompatibility' with the medium to be defoamed. A defoamer that is too compatible will not migrate into the foam lamella, rather into the complete coating system. In this case, the defoaming properties are minimal and the addidestroying the bubbles. Defoamers that are too incompatible, on the other hand, may lead to paint defects such as haze or craters. The selection of the proper defoamer can be characterized as a balancing act between compatibility and incompatibility (Fig. 7).

Of course, the optimum exists when proper defoaming without defects (haze, craters) is achieved. Due to the wide variety of paint systems just one defoamer cannot be optimal for all formulations. A range of various defoamer products is necessary to offer a suitable product for each purpose.

The fine tuning of the defoaming action can be achieved by the dosage: a higher amount of defoamer in general brings better defoaming, however it may tive may even stabilize foam instead of

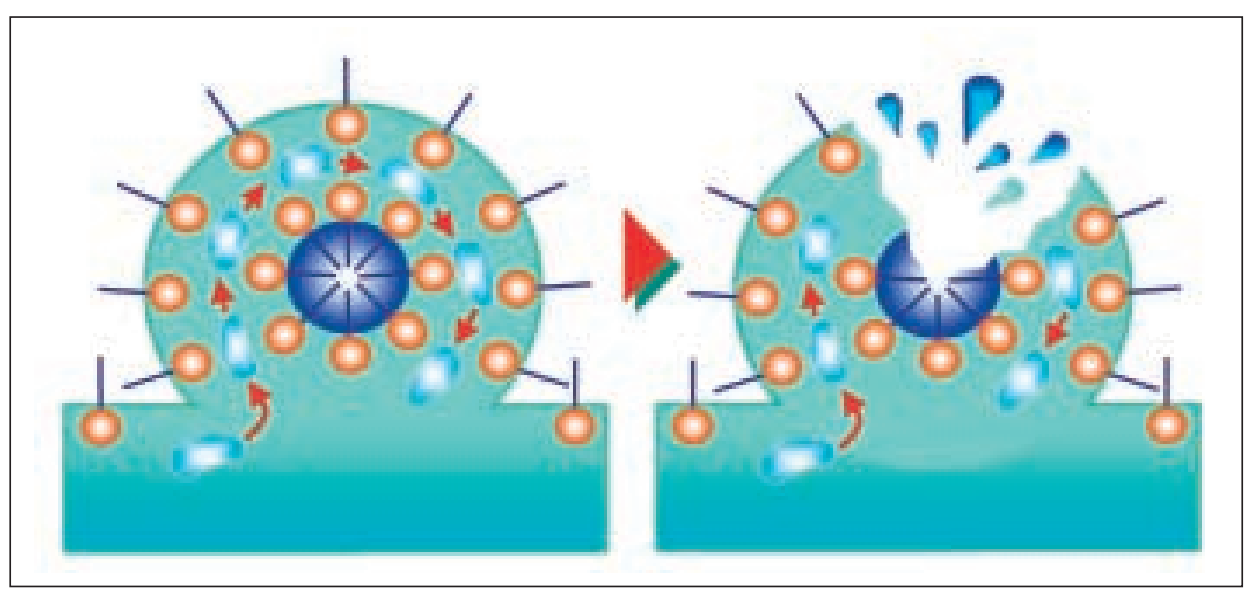

Fig. 5. Penetration of defoamer into the lamella of the foam bubble

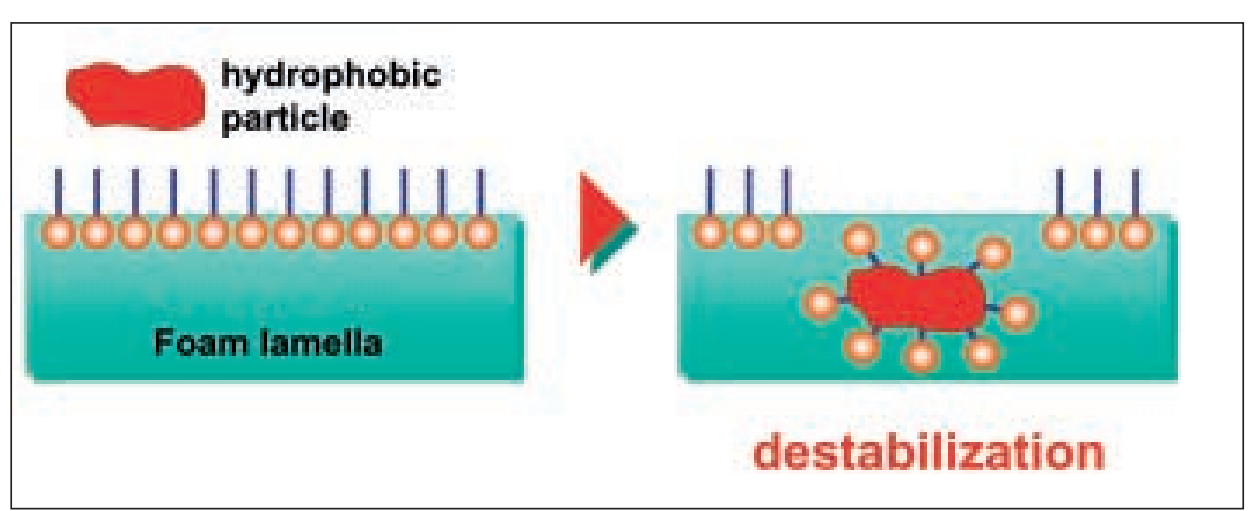

Fig. 6. Foam destabilization by hydrophobic particles increase defects or make them visible. A lower dosage avoids film defects but the defoaming action may not be sufficient.

Also the shear forces which are available during incorporation of the (incompatible) defoamer into the coating system can be a crucial factor. When the shear forces are not high enough the defoamer will not be homogeneously distributed in

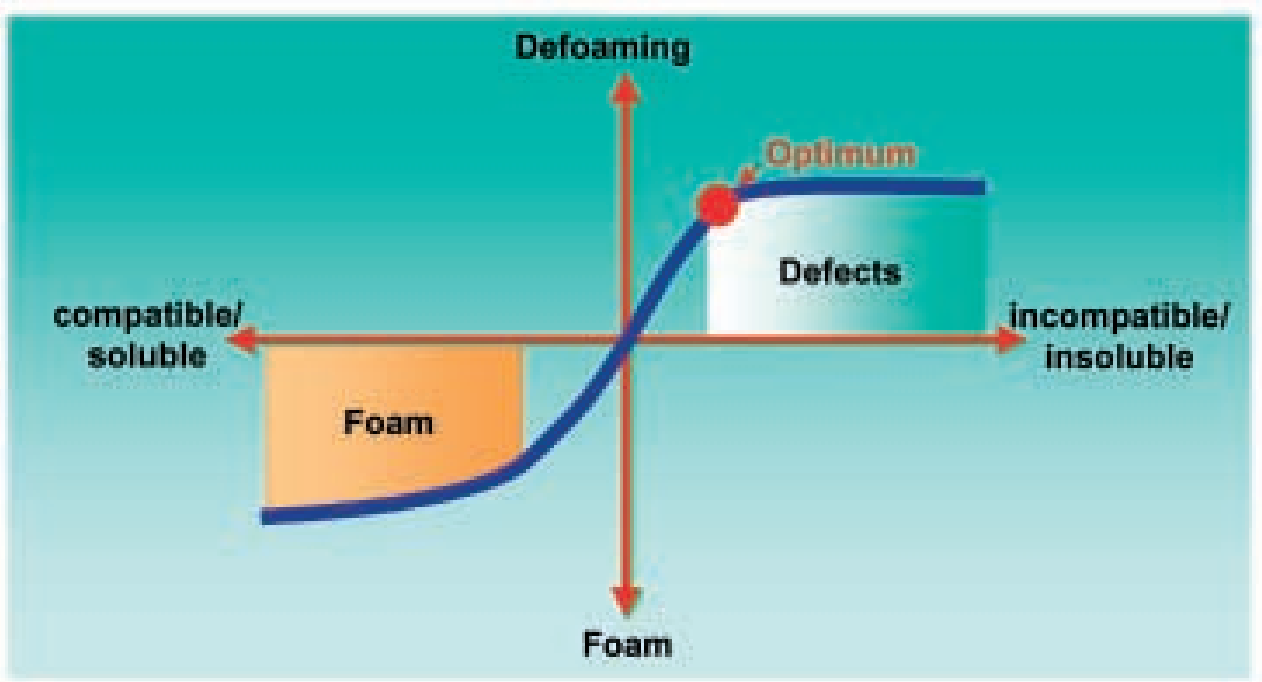

Fig. 7. Correlation between defoamer incompatibility and defoaming efficiency the system and craters can develop due to the locally high concentration of incompatible material. Very incompatible defoamers should be already added to the millbase whereas for more compatible products the lower shear forces during the let-down phase are sufficient.

\section{Chemistry of Defoamers}

The following discussion will focus upon the chemistry which is used for the production of defoamers for aqueous and solvent-based/solvent-free coating systems.

In contrast to the most other application fields in which defoamers are needed (metal treatment, food processing, polymer industry, textile industry) defoamers for the coatings industry are more or less complex formulations, which have to be especially designed for the respective binder system.

Defoamers for coatings can be categorized into the following groups:

- Mineral oil defoamers

- Silicone defoamers

- Silicone-free polymeric defoamers 


\subsection{Mineral Oil Defoamers}

Mineral oil defoamers are intended primarily for usage in flat and semi gloss emulsion paints and plasters. In higher quality aqueous industrial coatings, mineral oil defoamers are not suitable as they can cause surface defects like oil separation or gloss reduction of the final paint. Additionally, in solvent-based systems they should not be used because their spreading efficiency is not high enough.

A mineral oil defoamer is composed of approximately 85-95\% carrier oil and $1-3 \%$ hydrophobic particles. The remaining $5 \%$ are emulsifiers, biocides and other enhancing ingredients. For easier incorporation the mineral oil defoamer can be formulated as an emulsion in water.

As the carrier oil, aromatic or aliphatic mineral oils can be considered. Aromatic products are no longer employed since they may cause premature yellowing of the paint film and since they may represent physiological handling risks because of their high levels of polycyclic aromatic hydrocarbons. For some special applications like paints and inks with food contact medicinal white oils or vegetable oils can be used as carriers.

Hydrophobic particles also exhibit a decisive influence upon defoamer behavior. Hydrophobic fumed silicas are generally employed, or in some cases milled polyamide particles, waxes or metal soaps. These silica particles are produced by treatment of the particle surface with polydimethylsiloxanes or by reaction of the $\mathrm{OH}$-groups of the particles with organo silicon halides. Non-modified silica particles exhibit bad performance in most systems and are not recommended. Parti- cles of polyamide, waxes and so forth can be made by chilling processes or by milling.

In the last years significant progress has been made in the usage of polyurea particles. This polyurea is generated from liquid reaction partners in situ in the carrier oil, thus leading to a much finer distribution of the particles and a reduced separation tendency. This is valid for both mineral oil defoamers and silicone defoamers, which will be discussed later.

Because of the larger specific surface available, the adsorption capacity concerning surfactants is greater. This in turn assures optimal defoamer activity even after long-term storage of the finished coating.

The emulsifiers utilized in defoamers are required to disperse the particles within the carrier oil. In addition, they exert a positive influence upon ease of defoamer incorporation. Most commonly alkylphenol ethoxylates were used as emulsifiers in defoamers. Today mostly ethoxylates/propoxylates of linear or branched aliphatic alcohols are the right choice.

\subsection{Silicone Defoamers}

Silicone defoamers are liquids containing active ingredients (polysiloxanes) with exceptionally low surface tension. When one chooses a polysiloxane, chemical structure is the decisive point. For example, the relatively short-chain polysiloxanes (which are used as surface additives) can demonstrate foam stabilizing, rather than foam-destabilizing (defoaming) behavior. Whether or not a particular polysiloxane functions as a foam stabilizer or as a defoamer depends upon the product's compatibility and solubility in the liquid medium at hand; only selectively incompatible and insoluble polysiloxanes function as defoamers. Fig. 8 demonstrates this point in regard to the pure (unmodified) dimethylpolysiloxanes. The controlling factor here is the molecular weight or the chain length of the silicone. Lower molecular weight products function as foam stabilizers; higher molecular weight analogues are incompatible enough to create craters or fish-eyes; and finally, the higher molecular weight products (hammer finish silicones) are completely incompatible.

For this reason non-modified polydimethylsiloxanes are rarely utilized in the paint industry. The silicones for coatings contain organic modifications which give an excellent control over the degree of incompatibility of these materials.

The modifications of these silicones, that means the adjustment of the required 'selective incompatibility' is done essentially by reaction of $\mathrm{Si}-\mathrm{H}$-groups, which are incorporated by equilibration reaction into the polydimethylsiloxane chain and by variation of the molecular weight of the polydimethylsiloxane chain.

In this equilibration reaction (Scheme 1), catalyzed by sulphuric acid, bleaching earths or acidic fluoro compounds like trifluoro methane sulphonic acid, reactive $\mathrm{Si}-\mathrm{H}$-groups are inserted into the polydimethylsiloxane backbone.

The two most important reactions of $\mathrm{Si}-\mathrm{H}$-groups are the addition (Scheme 2) of allylic compounds (e.g. mono-allylic polyglycols, olefins) and the condensation (Scheme 3) of alcohols (e.g. monohydroxy-functional polyglycols, linear or branched aliphatic alcohols).
Fig. 8. Chemical structure of non-modified polydimethylsiloxane

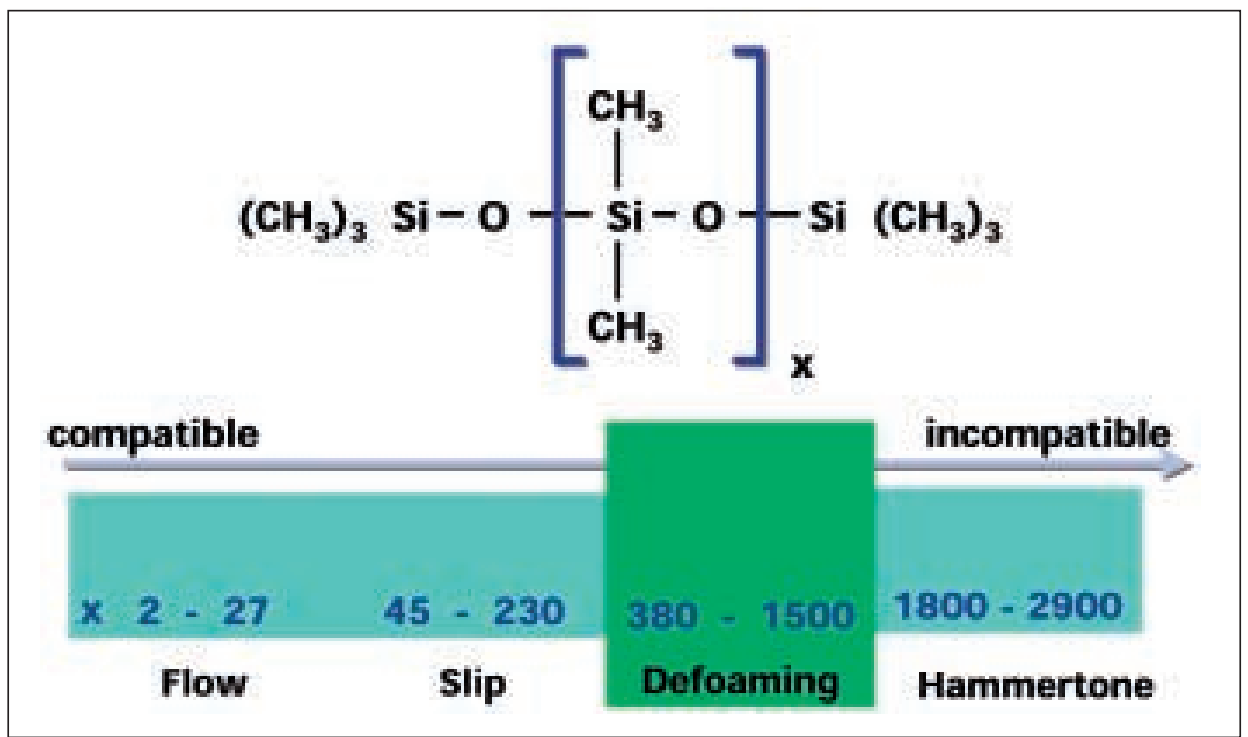



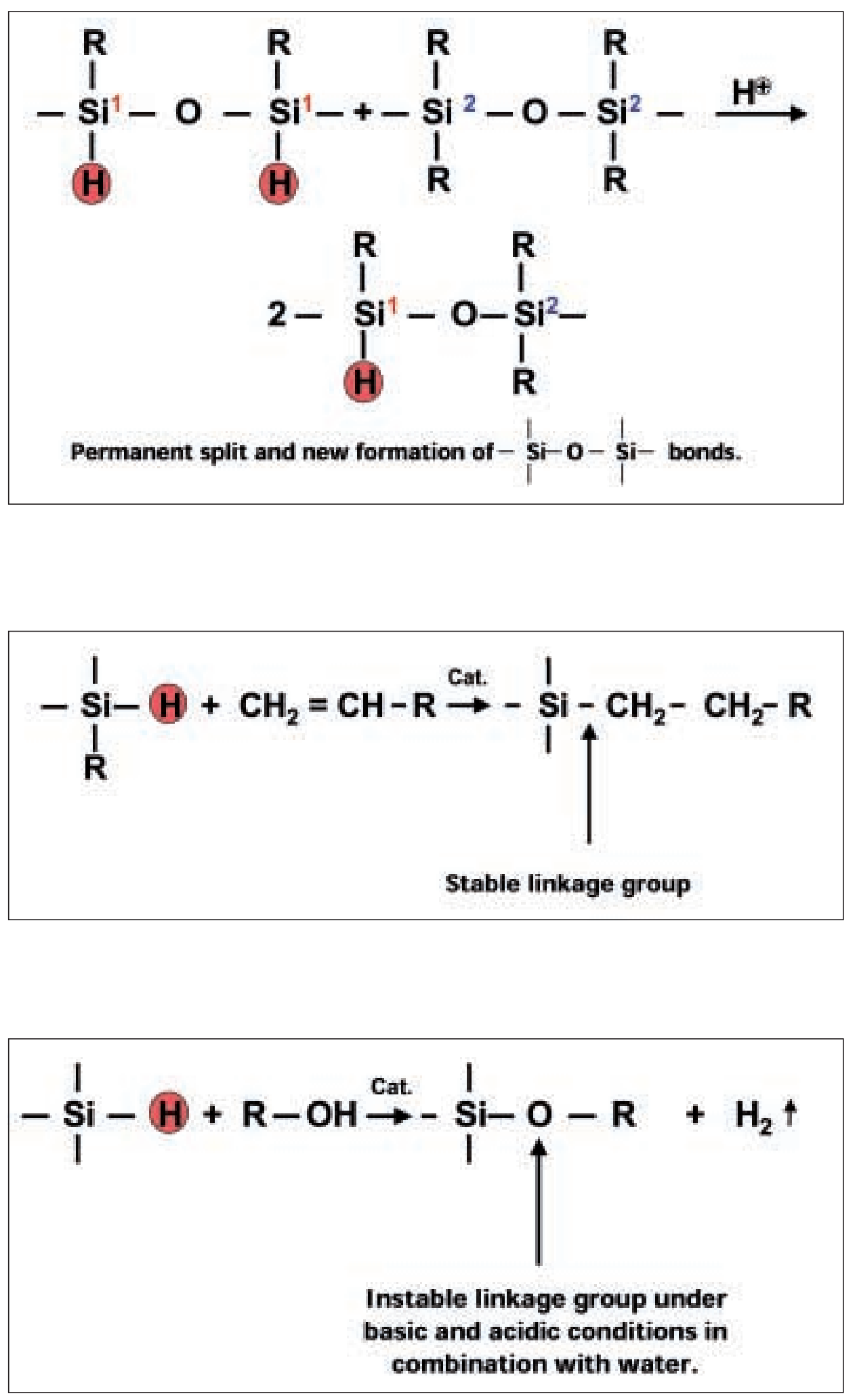

The addition of polyether chains increases hydrophilicity and, therefore, as a rule, increases compatibility in polar systems (Fig. 9). In place of the dimethylpolysiloxanes, methylalkylsiloxanes can also be employed.

The exchange of the second methyl group with a longer alkyl chain leads to higher silicone surface tension values. In general, this means a decrease in foam stabilization behavior. The general structure of such silicones is shown in Fig. 10.

Recent innovations include the introduction of defoamers with perfluorinated organic modifications, the so-called 'fluorosilicone defoamers'. These products distinguish themselves by their very low surface tensions and their strong
Scheme 1. Silicone chemistry: equilibration reaction

Scheme 2. Silicone chemistry: addition reaction

Scheme 3. Silicone chemistry: condensation reaction

defoaming behavior. These compounds, already effective in concentrations of the lower ppm range, do not cause crater or other side effects if they are incorporated into the paint system under high shear conditions. A schematic structure is shown in Fig. 11. 
Fig. 9. Chemical structure of polyether-modified dimethylpolysiloxanes

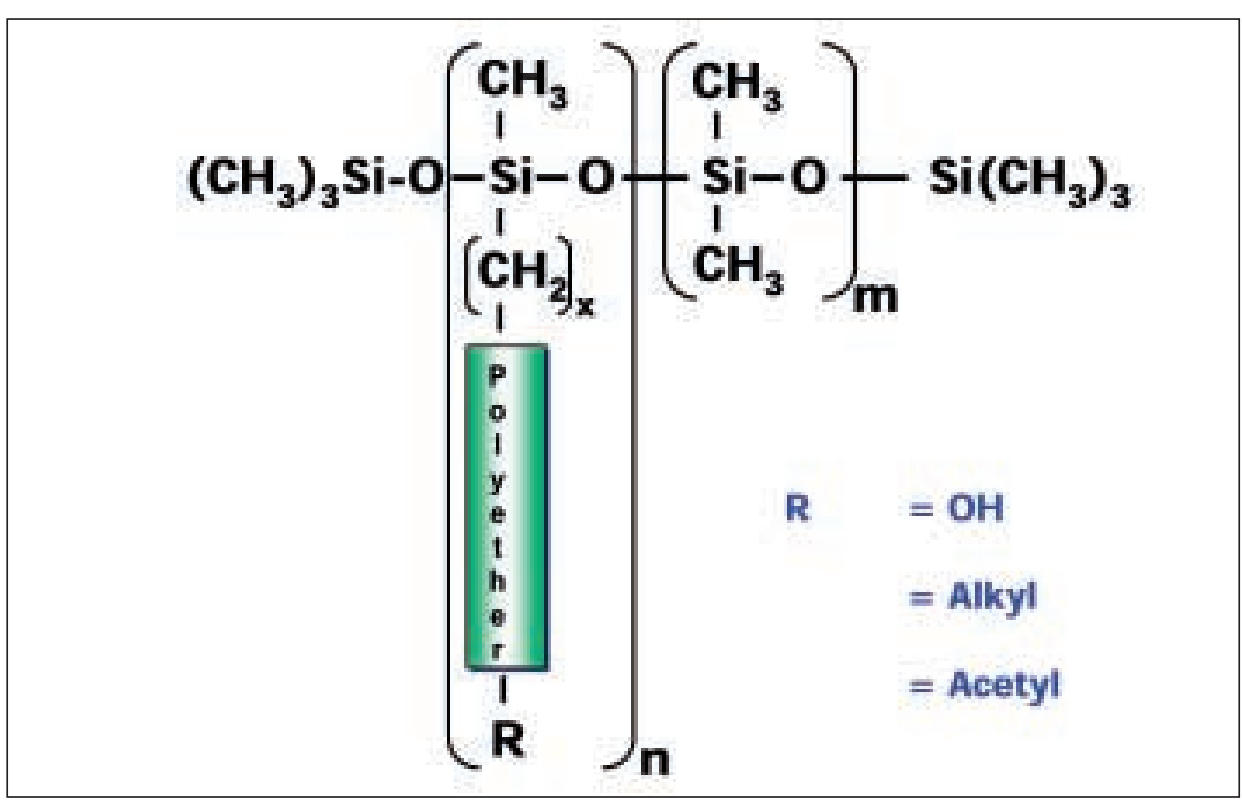

Fig. 10. Chemical structure of polyether-modified methylalkylpolysiloxanes

\subsection{Silicone Defoamers for Aqueous Coatings}

Silicone defoamers for aqueous systems are mostly emulsions of strongly hydrophobic silicone oils. Some silicones can be formulated with special polyglycols or other additives like e.g. emulsifiers, which support the defoaming behavior. Due to their silicone content silicone defoamers are more expensive than mineral oil defoamers and therefore designed for the utilization in high-quality coatings formulations. Silicone defoamers can also be combined with hydrophobic particles like polyureas and silica in order to improve silicone oil dispersibility and to improve defoaming performance. The primary advantage (as compared to mineral oil defoamers) is that they neither reduce gloss in high gloss systems, nor do they alter color acceptance in pigment concentrate systems.

The individual products vary according to not only the particular hydrophobic silicone oil employed, but also according to emulsifier type. Dependent upon which product is chosen, differences in crater susceptibility and in storage stability can be noted. However, in many instances, optimal incorporation of the defoamer via higher shear force - can result in completely crater-free coatings.
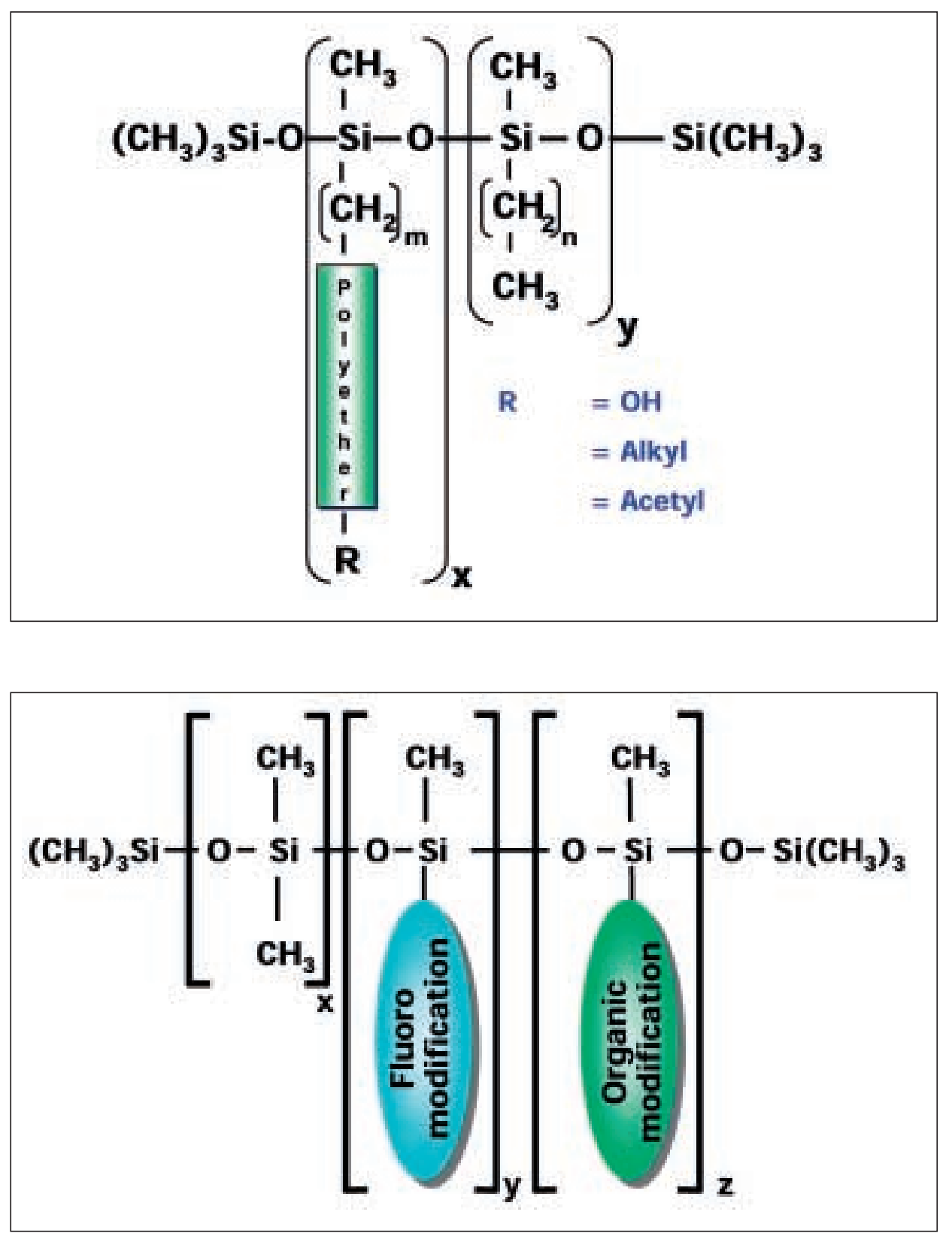

Fig. 11. Chemical structure of fluoro-modified polysiloxanes 
For utilization in wood and furniture coatings with low pigment levels or no pigments at all special defoamers are required. Because of the low viscosity of such systems no high shear forces are available. Therefore, these special defoamers exhibit greatly enhanced ease of incorporation and sometimes they can be used as a post additive in curtain coater systems without fear of side effects.

\subsection{Silicone Defoamers for Solvent- based Systems}

The above-mentioned fluoro-modified polysiloxanes have a broad application spectrum. They offer highly efficient defoaming even with minimal amounts of incompatible substances. Sometimes only amounts in the ppm range are needed to get the desired defoaming effect.

For these types of defoamers it is extraordinary important that they are incorporated under high shear forces for optimal, defect-free coatings.

\subsection{Silicone-free Polymeric Defoamers}

Not only can polysiloxanes be used as defoamers, but also other polymeric products can defoam through their selective incompatibility. To achieve the proper balance between 'compatibility' and 'incompatibility', one can intentionally modify polymeric polarity and molecular weight (molecular weight distribution).

With respect to exact correlations between defoamer behavior and the chemistry of the special polymeric defoamer compounds, there is unfortunately no published data. Detailed data are proprietary information.

Some polymers with high molecular weight with an acrylic backbone act both as defoamer and as flow or leveling agents. A well-known example in paint industry is the poly-n-butyl acrylate with a high molecular weight.

\subsection{Defoamers for UV Systems}

UV systems differ in polarity from binder to binder. Mostly polyurethane acrylates, epoxide acrylates or polyester acrylates are used, with or without water. Moreover defoamer properties in these systems are influenced by the kind and amount of reactive diluent and other parts of the formulation. Therefore suitable defoamers have to be chosen in test series. A theoretical prediction of the suitable defoamer is difficult. Normally modified silicones, fluorosilicones or polymeric defoamers, e.g. especially designed incompatible polyacrylates, are the right choice. In some cases low mole- cular weight alkoxylates are described to be effective. Due to the fact that UV systems are frequently non-pigmented (e.g. parquet lacquers) crater tendency but also film turbidity of the final coating must be taken care of when using defoamers.

\section{Selection Criteria and Test Methods}

Since defoaming action itself is the most important selection criterion for defoamers, many different test methods are employed. The fastest and easiest method is the evaluation of the defoamer in pure resin. In particular, air is incorporated into the resin as one observes how rapidly bubbles break (or more precisely, how quickly the foam volume is reduced). Nevertheless, an evaluation such as the above must be characterized as only a 'pre-test' since the final coating formulation contains numerous additional components which can also influence foam behavior. It is therefore mandatory that one perform defoamer tests in the final coating system itself.

In order to distinguish performance between the control system and the various defoamer test series, a reproducible method of incorporating air (or producing foam) is recommended. Since the objective is to achieve comparable results regardless of the method employed, then 'absolute' methods are not of importance. The main point is the production of as much or as little foam as necessary to differentiate the test results.

After air has been incorporated, then one can observe (1) the foam reduction over time and/or (2) the visually perceptible defects in the final applied paint film. Evaluation techniques include, for example, microscope examinations or weathering tests. It is often helpful to apply the foamed paint to a glass plate or a plastic sheet, thereby visually observing (with light passing through the plate) the film. In highly filled systems (such as plasters), density evaluation is most useful; the higher the density the better the defoaming.

Since great variations exist with regard to foam behavior in different coating systems, one cannot logically recommend any one standard method.

The coating should be tested approximately $24 \mathrm{~h}$ after defoamer incorporation. In addition, evaluations after storage (since defoamers can lose effectiveness over time) should be completed. Significant performance differences can be noted after only four weeks storage at $50^{\circ} \mathrm{C}$.
Since many defoamers contain hydrophobic particles, it is also recommended that one also observe the stability (separation tendency) of the defoamer itself. Otherwise, the homogeneous distribution of the raw material in the container cannot be guaranteed.

Defoamers are effective when they are insoluble in the medium to be defoamed and when they display a certain degree of selective incompatibility. As a result, the following potential side effects should be considered:

- Gloss decrease

- Haziness in clear systems

- Tendency toward crater formation

- Possible influence of intercoat adhesion

Whether or not a particular coating system exhibits foam problems is dependent not only upon the formulation itself, but also upon substrate conditions (e.g. porosity) and application parameters (e.g. spray pressure, nozzle size, paint flow rate, etc.). One should always attempt to include the most important application parameters in the test program. Such inclusion provides the only guarantee of obtaining both definitive and practical results.

Received: March 28, 2002

'Antifoams', in 'Encyclopedia of Polymer Science and Engineering', 2nd ed., vol. 2, Eds. H.F. Mark, N. Bikales, C.G. Overberger, G Menges, John Wiley \& Sons, 1985, p. 59-72.

'Foams and Foam Control', in 'Ullmann's Encyclopedia of Industrial Chemistry', vol. A 11, 5th ed., VCH, Weinheim, 1988.

'Defoaming: Theory and Industrial Applications', in 'Surfactant Science 45', Ed. P.R. Garrett, Marcel Dekker Inc., New York, 1993.

R.J. Pugh, 'Foam Breaking in Aqueous Systems', in 'Handbook of Applied Science and Colloid Chemistry', John Wiley \& Sons, 2001. R.J. Pugh, 'Foams and Foaming', in 'Handbook of Applied Science and Colloid Chemistry', John Wiley \& Sons, 2001.

P.R. Garrett, J. Davis, H.M. Rendall, 'An experimental study of the antifoam behavior of mixtures of a hydrocarbon oil and hydrophobic particles', Colloids and Surfaces A: Physicochemical and Engineering Aspects 1994, 85, 159-167. E.W. Orr, 'Part IV: Defoaming', in 'Performance Enhancement of Coatings', Hanser, Munich, 1998. 